\section{HEAVY FERMIONS}

\author{
Peter Fulde, Stuttgart
}

(Max-Planck-Institut für Festkörperforschung)

Over the past years, a new field has become established in metal physics which has already led to a number of interesting new phenomena. It deals with substances which have the characteristic feature that their low temperature thermodynamic properties are those of an ordering metal with very large electron masses. Generally the thermodynamics at low temperatures is determined by the quasiparticles of the system. These are excitations of the electronic system with energies $E \cong k_{\mathrm{B}} T$ where $T$ is the temperature and $k_{\mathrm{B}}$ is Boltzmann's constant. It is the mass of those quasiparticles which is found to be so large, in practice going up to more than 200 times the free electron mass $m_{\mathrm{e}}$. The quasiparticles (or quasiholes) are therefore almost as heavy as $\mu$ mesons. Because they are fermions, these systems are called heavy fermion systems and at present approximately 15 heavy (or medium heavy) systems are known. They are predominantly $\mathrm{Ce}$ and $\mathrm{U}$ compounds and their number is steadily increasing.

It is clear that the large quasiparticle mass must result from strong electron correlations (many body effects). When an electron moves through the system, its motion requires rearrangements of other electrons in order to reduce the Coulomb repulsions. The quasiparticle, which can be considered as the complex consisting of an electron and its rearrangement cloud, moves then much more slowly than a free electron of the same energy. It acts therefore like a particle with a large mass.

Before discussing some of the characteristic properties of heavy fermion systems, let us see how one became aware of them.

In 1975 the obervation was made ${ }^{1}$ ) that below $0.3 \mathrm{~K}$ the compound $\mathrm{CeAl}_{3}$ had a linear specific heat $C=\gamma T$ with a Sommerfeld coefficient $\gamma$ which is by three orders of magnitude larger than that of $\mathrm{Na}$ for example; $\gamma$ is a measure of the conduction electron density of states which in turn is proportional to the effective mass $m^{*}$. An increase in $\gamma$ therefore, must be interpreted as arising from an increase in the effective mass by nearly the same amount. The behaviour of $C(T)$ for $\mathrm{CeAl}_{3}$ is shown schematically in Fig. 1.

One notices that the linear behaviour in $T$ changes quickly as the temperature increases, and that at higher temperatures, $C(T)$ increases much more slowly. This indicates that the heavy quasiparticles exist only at temperatures $T<T^{*}$ where $T^{*}$ is of the order of a few Kelvin. For $T>T^{*}$ the quasiparticles lose their heavy masses and approach more and more the behaviour of free electrons. This is a characteristic feature of all heavy fermion systems and the rule is that the larger the low temperature $m^{*}$ is, the smaller is $T^{*}$.

Another characteristic property of metals is a temperature independent Pauli paramagnetism. Like the specific heat coefficient $\gamma$, the magnetic susceptibility $\chi$ is also proportional to the density of states and therefore to the effective mass of the quasiparticles.

In accordance with this, $\mathrm{CeAl}_{3}$ has a low temperature susceptibility which is constant and, by almost three orders of magnitude, larger than that of ordinary metals. The temperature independence of $\chi$ holds true though, only for $T<T^{*}$. For $T>T^{*}$, the susceptibility is Curie-like with a Curie constant as expected for a well localized 4 f-electron per Ce site in a $J=5 / 2$ multiplet. Therefore it appears that the magnetic moment of the $\mathrm{Ce}$ ions is quenched below $T^{*}$ which reminds one of the Kondo effect (see separate panel). Since the ions with Kondolike behaviour (i.e. the $\mathrm{Ce}$ ions) form a lattice, the heavy fermion $\mathrm{Ce}$ compounds are often also called "dense Kondo" or "Kondo lattice" systems.

The richness of the new physics opening up became clear after the surprising discovery of superconductivity in $\mathrm{Ce}$ $\left.\mathrm{Cu}_{2} \mathrm{Si}_{2}{ }^{2}\right)$. This compound has an effective mass of $m^{*}=220 \mathrm{~m}_{\mathrm{e}}$ below $T^{* 1} \cong 5 \mathrm{~K}$ and becomes superconducting at $T_{c}=0.6 \mathrm{~K}$. The jump in the specific heat at $T_{c}$ relates to the large specific heat coefficient $\gamma$ and provides direct proof that the heavy quasiparticles are responsible for Cooper pair formation.

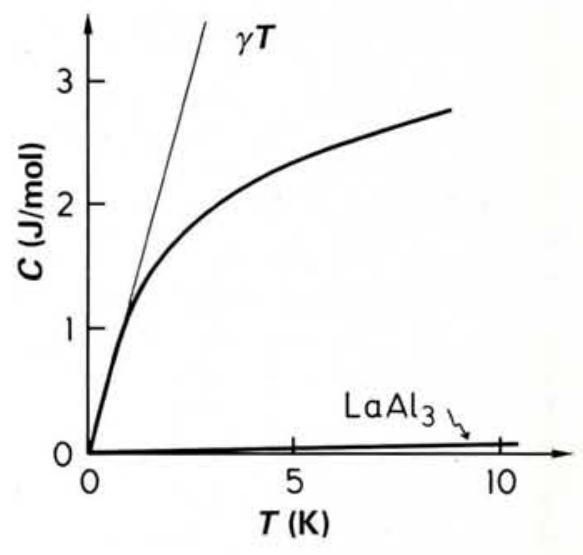

Schematic plot of the specific heat in $\mathrm{CeAl}_{3}$. The linear behaviour at low $T$ indicates a heavy Fermi liquid. For comparison, the curve for the related compound $\mathrm{LaAl}_{3}$ is also included.

It was some time before it was generally accepted that $\mathrm{CeCu}_{2} \mathrm{Si}_{2}$ is a superconductor because $\mathrm{Ce}$ ions were known to destroy superconductivity. An example is $\mathrm{Ce}$ dissolved in $\mathrm{LaAl}_{2}$ where the superconducting transition temperature is rapidly quenched as a function of the Ce concentration. This is due to the magnetic moments of the added $\mathrm{Ce}$ ions breaking up the Cooper pairs through their interaction with the conduction electrons.

In $\mathrm{CeCu}_{2} \mathrm{Si}_{2}$ the magnetic moments of the $\mathrm{Ce}$ ions are quenched at temperatures $T \leq T_{c}<<T^{*}$. The related compound $\mathrm{LaCu}_{2} \mathrm{Si}_{2}$ is not superconducting and has no unusual effective mass, yet the only difference relative to $\mathrm{CeCu}_{2} \mathrm{Si}_{2}$ is the $4 \mathrm{f}$-electron in the $\mathrm{Ce}$ ions. Therefore we must conclude that this $4 \mathrm{f}$-electron is not only responsible for the heavy fermion behaviour of $\mathrm{CeCu}_{2} \mathrm{Si}_{2}$ but also for generating superconductivity in it.

After superconductivity in $\mathrm{CeCu}_{2} \mathrm{Si}_{2}$ became an undeniable fact, a considerable increase in research activity followed as a result of which a number of other heavy fermion systems were found to be superconductive, notably $\mathrm{UBe}_{13}$ and $\mathrm{UPt}_{3}$. The experimental situation has been summarized in a recent review ${ }^{3}$ ).

The following questions have been intensively discussed in the literature:

(a) what is the interaction mechanism between the quasiparticles which leads to their mutual attraction and therefore Cooper pair formation?

(b) what kind of Cooper pairs do form, i.e., are we dealing with spin-singlet pairing as in ordinary superconductors or with spin-triplet pairing as in ${ }^{3} \mathrm{He}$ ?

From careful Josephson current measurements it seems certain by now that superconducting $\mathrm{CeCu}_{2} \mathrm{Si}_{2}$ is in a singlet pairing state. For the $U$ compounds the 
question is still undecided and further research is required. In any case the superconducting order parameter is expected to be anisotropic in momentum space, because the Fermi surface will be anisotropic in the heavy fermion systems.

Now that singlet pairing has been established in $\mathrm{CeCu}_{2} \mathrm{Si}_{2}$, there is little doubt that the mutual quasiparticle attraction leading to superconductivity is due to phonons in that substance. Again, for the $U$ compounds this is still an open question and it is conceivable that there the quasiparticle-phonon interaction is unimportant for the Cooper pair formation. Instead, the quasiparticle attraction would have to result from Coulomb and exchange interactions within the electronic system, with the ion positions kept fixed.

The quasiparticle-phonon interaction in heavy fermion systems is an interesting problem in itself. Consider the $\mathrm{Ce}$ compounds for example. For $T>T^{*}$ the interactions between the 4 f-electron and phonons are well understood (see e.g. Ref. 4). The $4 \mathrm{f}$-electron of a $\mathrm{Ce}$ ion can be considered as well localized and its eigenstates are described by conventional crystal field theory. Lattice vibrations (phonons) change this crystal field and therefore the eigenstates of the $4 \mathrm{f}$ electron. This provides a coupling mechanism between the two. Below $T^{*}$ the $4 \mathrm{f}$-electron must be considered as partially delocalized because a heavy fermion liquid forms. Then a deformation potential coupling between phonons and quasiparticles will take place as in any other metal. Only the coupling constant has to be determined anew. It turns out that it is determined by the pressure (or volume) dependence of $T^{*}$. This dependence can be measured experimentally and the quasiparticle-phonon interaction is therefore well characterized.

Let us now turn to a quantitative description of the quasiparticles. In particular we want to determine the dispersion $E(\mathbf{k})$, i.e. the energy bands of a quasiparticle. In the zero temperature limit, when the magnetic moment of the $\mathrm{Ce}$ ions has disappeared, we can characterize the ions of a heavy fermion system as in any other nonmagnetic metal by a scattering potential. An equivalent description is in terms of energy dependent phase shifts for different angular momenta $\ell$. The problem consists then in determining these phase shifts. From the above discussion it is clear that the $\ell=3$ or $\mathrm{f}$-phase shift at the Ce sites must play a distinct role because it is the delocalization of the $4 \mathrm{f}$-electrons which results in the heavy quasiparticles at low temperatu- res. Let us single out this phase shift and determine all the remaining phase shifts as in any other metal, i.e. by solving a one-particle Schrödinger equation with the potentials determined according to the local density approximation (LDA) to the density-functional method. Powerful linearized methods are available for performing such calculations even for large unit cells. This way one can determine all phase shifts with the exception of the one for $\ell=3$ at the $\mathrm{Ce}$ sites. That phase shift cannot be determined by making a local density approximation because the latter cannot describe the many-body features which lead to the Kondo effect. Therefore one makes for it the simplest possible ansatz by expanding it around the Fermi energy $\varepsilon_{\mathrm{F}}$ :

$$
\delta(\varepsilon)=\delta_{\mathrm{o}}+\alpha\left(\varepsilon-\varepsilon_{\mathrm{F}}\right) .
$$

For $\ell=3$ and $J=5 / 2$ there are $2 J+1=$ 6 scattering channels. Only those of the six channels which are known to form the crystal field ground state have a nonvanishing phase shift. They can be determined by inelastic neutron scattering experiments at $T>T^{*}$.

Eq. (1) contains two unknown parameters, i.e. $\delta_{0}$ and $\alpha$. The parameter $\delta_{0}$ is determined by a sum rule due to Friedel which relates it to the $4 \mathrm{f}$-electron occupancy $n_{f} \cong 1$. The other parameter $\alpha$ is adjusted so that in the end the right effective mass of the quasiparticles is obtained. One can set $\alpha=1 / T_{K}$ and define this way a Kondo temperature for the lattice. $T_{K}$ is then approximately equal to $T^{*}$ considered before.

When the Schrödinger equation is solved again, with the phase shift (1) taken into account, one finds the quasiparticle dispersion curves $E(\mathbf{k})$. The strong energy dependence of $\delta(\varepsilon)$ leads to a narrow resonance of width $k_{\mathrm{B}} T_{\mathrm{K}}$ at the Fermi surface which can describe the large effective masses of the quasiparticles.

Within the above computational scheme one can perform realistic calculations of quasiparticle-excitation bands. This has indeed been done for $\mathrm{CeCu}_{2} \mathrm{Si}_{2}{ }^{5}$ ). One interesting result is that close to the Fermi surface there are excitations corresponding to heavy masses as well as light masses. The latter do not contribute appreciably to the specific heat and susceptibility because of their low density of states, but it is conceivable that they play an important role in the transport properties.

One is dealing here with a new class of metals which have extraordinary anisotropies. For example, the superconducting properties should be different for the heavy and light quasiparticles and we expect that the light ones will cause

\section{THE KONDO EFFECT}

The Kondo effect was discovered in $1964^{1)}$ by J. Kondo who considered a magnetic ion embedded in a sea of conduction electrons with which it interacted through an antiferromagnetic exchange interaction:

$$
H_{\text {int }}=J_{\text {ex }} \mathbf{s}(0) \mathbf{S} \text {. }
$$

Here $\mathbf{s}(0)$ is the conduction electron spin at the site of the magnetic ion which has a spin $\mathbf{S}$. He found that due to the internal degrees of freedom of the ion (it can flip its spin) a perturbation expansion in terms of $H_{\text {int }}$ breaks down at low temperatures. The interacting magnetic ion-conduction electron system becomes an interesting many-body problem which requires complex theoretical methods for its solution. Exact solutions have been found only recently after many years of research (see e.g. Ref. 2). The magnetic susceptibility $\chi$ of the magnetic ion was shown to change from a Curie-like behaviour at high temperatures to saturation in the limit $T \rightarrow 0$. The cross-over occurs at a temperature $T_{\mathrm{K}}$, the Kondo temperature. In the low temperature limit $\chi=\pi / T_{\mathrm{K}}$. A quasiparticle description of the low temperature properties of the single-ion Kondo problem has been given ${ }^{3}$ ).

1. Kondo J., Progr. Theor. Phys. 32 (1964) 37.

2. Andrei N., Furuya K. and Lowenstein J.H., Rev. Mod. Phys. 55 (1983) 331; Tsvelick A.M. and Wiegmann P.B., Adv. Phys. 32 (1983) 453.

3. Nozières P.A., J. Low Temp. Phys. 17 (1974) 31

a vanishing energy gap in the singleparticle tunnelling density of states. Presumably there will be many more investigations of this topic in the future.

The heavy quasiparticle excitations are almost entirely of $f$-character. However, the narrow bands close to the Fermi energy do not imply in any way a single-particle approximation for an $\mathrm{f}$ electron wave function. The contrary is true. Photoelectron spectroscopy data demonstrate that it takes approximately $2 \mathrm{eV}$ in order to knock out an f-electron from a $\mathrm{Ce}$ ion in $\mathrm{CeCu}_{2} \mathrm{Si}_{2}$. Yet, with respect to the low temperature thermodynamic properties, the f-electrons show up right at the Fermi surface. There is no contradiction though between these two facts, because in a strongly correlated system high frequency and low frequency excitations behave very differently.

In conclusion one may state that the field of heavy fermions or almost localized electrons has started to develop into a 
fruitful field of research. Its present state is described in Ref. 5 which we recommend for further consultation.

\section{REFERENCES}

1. Andres K., Graebner J.E. and Ott H.R., Phys. Rev. Lett. 35 (1975) 1779.

2. Steglich F., Aarts J., Bredl C.D., Lieke W., Meschede D., FranzW. and Schäfer H., Phys. Rev. Lett. 43 (1979) 1892.

3. Stewart G.R., Rev. Mod. Phys. 56 (1984) 755.

4. Lüthi B., Chapter 4 in Dynamical Properties of Solids, Vol. 3, Eds. G.K. Horton and A.A. Maradudin, (North-Holland, Amsterdam) 1980

5. Proceedings of the "International Conference on Valence Fluctuations", Eds. E. Müller-Hartmann, B. Rhoden and D. Wohlleben, J. Mag. Mag. Mat. 47 and 48 (1985).

\section{JPS Collaboration}

Under the terms of an agreement concluded with the Physical Society of Japan (JPS), members of JPS may join the EPS as Category 4d) members (members of a Collaborating Society) on payment of a membership fee (for 1986) of Sw.Frs. 55. - (US\$26.-). In addition, members of JPS may submit papers to EPS meetings on the same conditions as EPS members, and will be entitled to pay the same registration fees for EPS meetings as members of EPS member societies.

In return, Individual Ordinary Members of EPS may submit papers to JPS meetings under the same conditions as JPS members, pay the same registration fees and may subscribe to the Journal of the Physical Society of Japan at the same rates as JPS members. Subscriptions for 1986 (vol. 55) should be addressed by 31 December 1985 to:

The Physical Society of Japan

Room 211, Kikai-Shinko Blg.

3-5-8 Shiba-Koen, Minato-ku

Tokyo 105, Japan

EPS Divisions, Sections and Group

Astronomy and Astrophysics Division Solar Physics Section

Atomic and Molecular Physics Division Atomic Spectroscopy Section Chemical Physics

Electronic and Atomic Collisions Molecular Physics

Computational Physics Group

Condensed Matter Division

Liquids Section

Low Temperature Physics Section

Macromolecular Physics

Magnetism

Metal Physics

Semiconductors and Insulators

Surfaces and Interfaces

High Energy \& Particle Physics Division

Nuclear Physics Division

Optics Division

Optics Division
Plasma Physics Division

Quantum Electronics Division

\section{EPS Lecturer 1985/1986}

The EPS Lecturer for $1985 / 86$ is Professor H. Haken of the University of Stuttgart. $\mathrm{He}$ is well known especially for his original work on "Synergetics"* but he has also made basic contributions to the quantum field theory of the solid state, to statistical physics and to quantum optics. Professor Haken's lecture tour will consist of two parts: from 20 October until 7 November 1985 he will visit three Scandinavian countries and England, and then next year he will spend two-three weeks in January/ February travelling through France, Portugal, Spain, Italy and Israel.

He will be presenting three lectures:

1. Synergetics, an overview

2. Theory of non-equilibrium phase transitions, slaving principle and order parameters

3. Pattern formation in systems far from thermal equilibrium.

\section{Programme of the First Part}

\begin{tabular}{|c|c|c|c|c|c|}
\hline & Date, 1985 & Location & Time & Lecture & Contact \\
\hline \multirow[t]{10}{*}{ Oct. } & 21 & Helsinki & 14.30 & 1 & S. Stenholm \\
\hline & 22 & Helsinki & $10: 30$ & 3 & S. Stenholm \\
\hline & 23 & Turku & $14: 15$ & 1 & M. Punkkinen \\
\hline & 24 & Turku & $10: 15$ & 2 & M. Punkkinen \\
\hline & 25 & Stockholm & $10: 00$ & 1 & B. Nagel \\
\hline & 25 & Stockholm & $14: 00$ & 3 & B. Nagel \\
\hline & 28 & Göteborg & $15: 15$ & 3 & B. Lundqvist \\
\hline & 29 & Göteborg & $15: 15$ & 1 & B. Lundqvist \\
\hline & 30 & Lund & $15: 30$ & 1 & B.E. Svensson \\
\hline & 31 & Lund & $10: 30$ & 3 & B.E. Svensson \\
\hline \multirow[t]{5}{*}{ Nov. } & 1 & Lyngby & $15: 00$ & 1 & P. Christianssen \\
\hline & 4 & Bristol & $14: 15$ & 3 & R.G. Chambers \\
\hline & 4 & Bristol & $17: 00$ & 1 & R.G. Chambers \\
\hline & 6 & Birmingham & $17: 00$ & 1 & W.F. Vinen \\
\hline & 6 or 7 & Birmingham & $14: 15$ & 2 & W.F. Vinen \\
\hline
\end{tabular}

The programme of the second part will be published in the November/December issue of Europhysics News.

* see for example Europhysics News 7 (1976) 7/8, 9.

Subscription fee to Europe is as follows:

Surface mail: $\quad$ Yen 13400

Airmail: $\quad$ Yen 38400

Microfilm (airmail): Yen 13400

In his concluding message to EPS, the outgoing President of JPS, Prof. Hiroshi Kamimura (now succeeded by Professor Yoshihiko Ichikawa) referred to the JPS "looking forward to a mutually beneficial exchange".

Europhysics News is the official journal of the European Physical Society which comprises 29 National Societies, Academies and Group, over 4000 Individual Members and 71 Associate Members. Governing bodies of EPS are the General Meeting, Council and an elected Executive Committee responsible for detailed policy. EPS promotes the collaboration of physicists throughout Europe, organising and harmonising conferences and publications, improving physics education, encouraging physics applications, awarding scholarships to sponsored schools in Erice. EPS publishes in addition to EN, Europhysics Conference Abstracts, E. Ed. News and, in collaboration with The Institute of Physics (UK). the European Journal of Physics. Individual Members receive EN free of charge (price to institutions: Sw.Fr. $90 .-1 a)$, rebates on the price of many publications and on conference fees. Annual EPS membership fee for 1986: Individual Members belonging to an EPS member society is: Sw.Fr. 44.; independent members: Sw.Fr. 132-; members of a Collaborating Society, e.g. the American Physical Society: Sw.Fr. 55.- (\$ 26).

\section{New Associate Member}

The latest organisation to become an Associate Member of EPS is:

\section{S.A.B.C.A. - Société Anonyme Belge de Construction Aéronautique 1470 Chaussée de Haecht B - 1130 Brussels}

\section{Editor: E.N. Shaw \\ Meetings Compilation: W.S. Newman \\ Editorial Board: \\ K. Appert, A. Baratoff, B. Jacrot, \\ G.R. Macleod, M. Mayor, J. Muller}

Editorial and Advertising Office at the EPS Secretariat

Address: EUROPEAN PHYSICAL SOCIETY P.O. Box 69 , $\mathrm{CH}-1213$ Petit-Lancy 2

Switzerland

Telephone: Geneva (22) 931130

Telex : $\mathbf{4 2 3} \mathbf{4 5 5}$ dema ch

Cables: europhys genève

Printed by: Pfirter frères sa $\mathrm{CH}-1213$ Petit-Lancy/Switzerland 